\title{
Connected Lie Groupoids are Internally Connected and Integral Complete in Synthetic Differential Geometry
}

Matthew BURKE

4 River Court, Ferry Lane, Cambridge CB4 1NU, UK

E-mail: matthew.burke@cantab.net

URL: http://www.mwpb.uk

Received June 29, 2016, in final form January 13, 2017; Published online January 24, 2017

https://doi.org/10.3842/SIGMA.2017.007

\begin{abstract}
We extend some fundamental definitions and constructions in the established generalisation of Lie theory involving Lie groupoids by reformulating them in terms of groupoids internal to a well-adapted model of synthetic differential geometry. In particular we define internal counterparts of the definitions of source path and source simply connected groupoid and the integration of $A$-paths. The main results of this paper show that if a classical Hausdorff Lie groupoid satisfies one of the classical connectedness conditions it also satisfies its internal counterpart.
\end{abstract}

Key words: Lie theory; Lie groupoid; Lie algebroid; category theory; synthetic differential geometry; intuitionistic logic

2010 Mathematics Subject Classification: 22E60; 22E65; 03F55; 18B25; 18B40

\section{Introduction}

In classical Lie theory we use a formal group law to represent the analytic approximation of a Lie group. Recall that an $n$-dimensional formal group law $F$ is an $n$-tuple of power series in the variables $X_{1}, \ldots, X_{n} ; Y_{1}, \ldots, Y_{n}$ with coefficients in $\mathbb{R}$ such that the equalities

$$
F(\vec{X}, \overrightarrow{0})=\vec{X}, \quad F(\overrightarrow{0}, \vec{Y})=\vec{Y} \quad \text { and } \quad F(F(\vec{X}, \vec{Y}), \vec{Z})=F(\vec{X}, F(\vec{Y}, \vec{Z}))
$$

hold. In fact there is an equivalence of categories

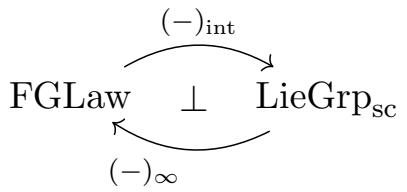

between the category FGLaw of formal group laws and LieGrp sc $_{\text {of }}$ simply connected Lie groups. The functor $(-)_{\infty}$ is obtained by considering the Taylor expansion of the multiplication of the Lie group close to the identity element. For more details see Theorem 3 of Section V.6 and Theorem 2 of Section V.8 of Part 2 in [26]. The functor $(-)_{\text {int }}$ therefore extends local data to global data and involves finding solutions to smooth time-dependent left-invariant vector fields.

In [5] we generalise the underlying adjunction of (1.1) to an adjunction

$$
\operatorname{Cat}_{\infty}(\mathcal{E}) \overbrace{(-)_{\infty}}^{\stackrel{i}{\perp}} \operatorname{Cat}(\mathcal{E}) \overbrace{\stackrel{+}{\perp}<}^{(-)_{\text {int }}} \operatorname{Catint}_{j}(\mathcal{E})
$$


between two subcategories of the category of internal categories in a topos $\mathcal{E}$. When considered together, this paper and [5] not only provide a more concise exposition of the thesis [4] but also contain several stronger results. The main improvement over [4] contained in this paper is the extension of the results about internal connectedness conditions from groups to groupoids.

In [5] we prove the result analogous to Lie's second theorem in this context: when we apply the appropriate connectedness conditions (described in Section 1.3) the functor $(-)_{\infty} j$ is full and faithful. In this paper we justify the work in [5] by describing the relationship between (1.1) and (1.2) in the case that $\mathcal{E}$ is a well-adapted model of synthetic differential geometry (see Section 1.1). This is carried out in Section 4.2 where we show that when we restrict $\operatorname{Cat}(\mathcal{E})$ to the full subcategory on the classical Lie groups, the functor $(-)_{\infty}$ coincides with the formal group law construction described in the Introduction of [14].

In addition we relate the adjunction (1.2) to the established generalisation of Lie theory involving Lie algebroids and Lie groupoids. (See for instance [21].) A Lie groupoid is a groupoid in the category of smooth paracompact manifolds such that the source and target maps are submersions. A Lie algebroid is a vector bundle $A \rightarrow M$ together with a bundle homomorphism $\rho: A \rightarrow T M$ such that the space of sections $\Gamma(A)$ is a Lie algebra satisfying the following Leibniz law: for all $X, Y \in \Gamma(A)$ and $f \in C^{\infty}(M)$ the equality

$$
[X, f Y]=\rho(X)(f) \cdot Y+f[X, Y]
$$

holds. In the theory of Lie groupoids and Lie algebroids we have have a functor

$$
\text { LieAlgd } \stackrel{(-)_{\infty}}{\longleftarrow} \text { LieGpd, }
$$

which is full and faithful but not essentially surjective. Any Lie algebroid integrates to a topological groupoid, its Weinstein groupoid [6], but there can be obstructions to putting a smooth Hausdorff structure on it. For instance see [8] for a Lie algebroid whose Weinstein groupoid is a smooth but non-Hausdorff Lie groupoid and [1] for a Lie algebroid whose Weinstein groupoid is non-smooth. Therefore when dealing with integrability (for instance in [6]) the category of smooth manifolds is enlarged to include non-Hausdorff manifolds. Furthermore in [27] Tseng and Zhu show that the category of differentiable stacks contains all Weinstein groupoids whilst still retaining the concept of tangent vectors. Another approach, pursued in [5], is to use the theory of synthetic differential geometry where the Weinstein groupoid construction is always possible.

In the process of reformulating the theory of Lie groupoids and Lie algebroids in [5] it is necessary to use internal versions of certain conditions describing connectedness and solutions to a specific type of vector field. In this paper we will justify these assumptions by showing that all classical Hausdorff Lie groupoids satisfy these stronger conditions. Since the Weinstein groupoid construction is always possible in $\mathcal{E}$ the assumption that our groupoids are Hausdorff does not affect the part of the theory dealing with integrability, only the extent to which the conditions involving completeness and solutions to vector fields generalise the classical ones. So unless otherwise stated all Lie groupoids in this paper will have Hausdorff arrow space.

\subsection{Synthetic differential geometry}

In synthetic differential geometry we replace the category Man of smooth paracompact Hausdorff manifolds with a certain kind of Grothendieck topos $\mathcal{E}$ called a well-adapted model of synthetic differential geometry. In this section we sketch the axioms of a well-adapted model of synthetic differential geometry and recall a few key properties.

Firstly there is a full and faithful embedding $\iota:$ Man $\longmapsto \mathcal{E}$ and therefore a ring $R=\iota \mathbb{R}$ in $\mathcal{E}$. In addition we have the objects

$$
D_{k}=\left\{x \in R: x^{k+1}=0\right\},
$$


which are not terminal. In fact the fundamental Kock-Lawvere axiom holds: the arrow $\alpha: R^{k+1}$ $\rightarrow R^{D_{k}}$ defined by

$$
\left(a_{0}, a_{1}, \ldots, a_{k}\right) \mapsto\left(d \mapsto a_{0}+a_{1} d+\cdots+a_{k} d^{k}\right)
$$

is an isomorphism. A set of non-classical objects that will be useful in the sequel are the Weil spectra which are of the following form:

$$
\operatorname{Spec}(\text { Weil })=\left\{\left(x_{1}, \ldots, x_{n}\right): \bigwedge_{i=1}^{n}\left(x_{i}^{k_{i}}=0\right) \wedge \bigwedge_{j=1}^{m}\left(p_{j}=0\right)\right\},
$$

where $n, m \in \mathbb{N}_{\geq 0}, k_{i} \in \mathbb{N}_{>0}$ and the $p_{j}$ are polynomials in the $x_{i}$. We write $D_{\infty}=\bigcup_{i} D_{i}$ and $D=D_{1}$.

The following is Definition 3.1 in Part III of [17].

Definition 1.1. A pair of maps $f_{i}: M_{i} \rightarrow N(i=1,2)$ in Man with common codomain are said to be transversal to each other iff for each pair of points $x_{1} \in M_{1}, x_{2} \in M_{2}$ with $f_{1}\left(x_{1}\right)=f_{2}\left(x_{2}\right)$ (=y say), the images of $\left(d f_{i}\right)_{x_{i}}(i=1,2)$ jointly span $T_{y} N$ as a vector space.

Definition 1.2. A topos $\mathcal{E}$ together with a full and faithful embedding $\iota:$ Man $\rightarrow \mathcal{E}$ is a welladapted model of synthetic differential geometry iff

- the functor $\iota$ preserves transversal pullbacks,

- the functor $\iota$ preserves the terminal object,

- the functor $\iota$ sends arbitrary open covers in Man to jointly epimorphic families in $\mathcal{E}$,

- the internal ring $\iota(\mathbb{R})$ satisfies the Kock-Lawvere axiom,

- for all Weil spectra $D_{W}$ the functor $(-)^{D_{W}}: \mathcal{E} \rightarrow \mathcal{E}$ preserves all colimits.

Remark 1.3. Since $\iota:$ Man $\rightarrow \mathcal{E}$ preserves transversal pullbacks it determines an embedding of $\operatorname{LieGpd}_{H}$ into $\operatorname{Grpd}(\mathcal{E})$. Here we have written $\operatorname{LieGpd}_{H}$ for the subcategory of LieGpd consisting of the groupoids that have Hausdorff arrow space.

Remark 1.4. If $M$ is a smooth manifold then we will often abuse notation by writing $M$ to denote the object $\iota(M)$ in the well-adapted model.

Using the Kock-Lawvere axiom we can show that $\iota(T M) \cong M^{D}$ as vector bundles over $M$ and that the Lie bracket corresponds to an infinitesimal commutator. For more detail see [17]. Furthermore in Section 4.2 we show that formal group laws correspond to groups of the form $\left(D_{\infty}^{n}, \mu\right)$.

\subsection{Smooth affine schemes and the Dubuc topos}

In Section 2.7 we will need a more detailed description of the coverage that generates the topos $\mathcal{E}$. Hence in that section we will work in a well-adapted model of synthetic differential geometry called the Dubuc topos. In this section we briefly sketch the essential features of the Dubuc topos and refer to [10] for more details. Note that this means that the results of Section 2.7 hold for all the well-adapted models generated by a site contained in the Dubuc site. For instance by referring to Appendix 2 of [23] we see that our results hold for the Cahiers topos (see [9]) and the classifying topos of local Archimedean $C^{\infty}$-rings (see Appendix 2 of [23]).

In addition in Section 4.5 it will be convenient to know that every representable object is a subobject of $R^{n}$ for some $n \in \mathbb{N}$. Therefore in that section we will work in any well-adapted model $\mathcal{E}$ that is generated by a subcanonical site whose underlying category is a full subcategory of the category of affine $C^{\infty}$-schemes as defined below. In particular this means that the results of Section 4.5 hold for the Dubuc topos. 
Definition 1.5. The category $\mathcal{C}$ of affine $C^{\infty}$-schemes has as objects pairs $[n, I]$ where $n \in \mathbb{N}$ and $I$ is a finitely generated ideal of $C^{\infty}\left(\mathbb{R}^{n}, \mathbb{R}\right)$. The arrows

$$
[n, I] \stackrel{f}{\rightarrow}[m, J]
$$

are equivalence classes of smooth functions $f \in C^{\infty}\left(\mathbb{R}^{n}, \mathbb{R}^{m}\right)$ such that

- we identify $f \sim g$ iff $f \equiv g(\bmod I)$,

- for all $j \in J$ we have $j f \sim 0$.

Now we define a slight generalisation of the notion of open set. Using these open sets we define the Dubuc coverage by using inverse images of smooth functions.

Definition 1.6. The open subobject $U$ of $[n, I]$ defined by $\chi_{U}: \mathbb{R}^{n} \rightarrow \mathbb{R}$ is the subobject

$$
\left[n+1,\left(I, \chi_{U} \cdot X_{n+1}-1\right)\right] \stackrel{\text { proj }}{\longrightarrow}[n, I],
$$

which intuitively corresponds to the subset $\chi_{U}^{-1}(\mathbb{R}-\{0\}) \cap[n, I]$. The Dubuc coverage $\mathcal{J}$ consists of the families of open subobjects

$$
\left(U_{i} \longmapsto[n, I]\right)_{i \in I}
$$

that are jointly surjective.

The site that we use to generate the Dubuc topos is the full subcategory of the category of affine $C^{\infty}$-schemes on the germ-determined schemes which are defined as follows:

Definition 1.7. For a smooth function $f: \mathbb{R}^{n} \rightarrow \mathbb{R}$ we write $\mathbf{g}_{x}(f)$ for the equivalence class of functions that is the germ of $f$ at $x \in \mathbb{R}^{n}$ and $G_{x}$ for the ring of germs of smooth functions at $x$. For an ideal of smooth functions $I \triangleleft C^{\infty}\left(\mathbb{R}^{n}, \mathbb{R}\right)$ we write $Z(I)$ for the zero-set of $I$ and

$$
\mathbf{g}_{x}(I)=\left\{\Sigma_{j=1}^{k} r_{j} \mathbf{g}_{x}\left(\phi_{j}\right):\left(r_{j} \in G_{x}\right) \wedge\left(\phi_{j} \in I\right)\right\}
$$

for the ideal generated by germs of elements of $I$. Then a scheme $[n, I]$ is germ-determined iff

$$
\forall g \in C^{\infty}\left(\mathbb{R}^{n}, \mathbb{R}\right), \quad\left(\forall x \in Z(I), \mathbf{g}_{x}(g) \in \mathbf{g}_{x}(I)\right) \Longrightarrow g \in I .
$$

We denote by $\mathcal{C}_{\text {germ }} \subset \mathcal{C}$ the full subcategory on the objects that are germ-determined. The Dubuc topos is the Grothendieck topos generated by taking sheaves on the site $\left(\mathcal{C}_{\text {germ }}, \mathcal{J}\right)$ where $\mathcal{J}$ is the Dubuc coverage.

\subsection{Internal connectedness}

In classical Lie theory we study how much of the data in a Lie groupoid can be recovered from the subset of this data that is infinitely close to the identity arrows of the Lie groupoid. Since global features such as connectedness cannot be captured by the infinitesimal arrows we need to restrict our attention to Lie groupoids that are source path and source simply connected.

We say that a Lie groupoid $\mathbb{G}$ with arrow space $G$ and object space $M$ is source path/source simply connected iff all of its source fibres are path/simply connected. Let $\mathbb{I}$ be the pair groupoid on the unit interval $I$ that has precisely one invertible arrow between each pair of elements of $I$. Then it is easy to see that the global sections of the object $\mathbb{G}^{\mathbb{I}}=\operatorname{Grpd}(\mathcal{E})(\mathbb{I}, \mathbb{G})$ in $\mathcal{E}$ are equivalent to arrows $I \rightarrow G$ in $\mathcal{E}$ that are source constant and start at an identity element of $G$. Therefore $\mathbb{G}$ is source path connected iff

$$
\Gamma\left(\mathbb{G}^{\mathbb{I}}\right) \stackrel{\Gamma\left(\mathbb{G}^{\iota} \mathbb{I}\right)}{\longrightarrow} \Gamma\left(\mathbb{G}^{\partial \mathbb{I}}\right)
$$


is an epimorphism in Set. We have written $\Gamma$ for the global sections functor and $\iota_{\mathbb{I}}: \partial \mathbb{I} \rightarrow \mathbb{I}$ for the full subcategory that is the pair groupoid on the boundary of $I$. In this case $\iota_{\mathbb{I}}$ is simply the inclusion of the long arrow $(0,1): \mathbf{2} \rightarrow \mathbb{I}$. Similarly $\mathbb{G}$ is source simply connected iff it is source path connected and

$$
\Gamma\left(\mathbb{G}^{\mathbb{I}^{2}}\right) \stackrel{\Gamma\left(\mathbb{G}^{\iota} \mathbb{\mathbb { L }}^{2}\right)}{\longrightarrow} \Gamma\left(\mathbb{G}^{\partial \mathbb{I}^{2}}\right)
$$

is an epimorphism in Set. We have written $\iota_{\mathbb{I}^{2}}: \partial \mathbb{I}^{2} \rightarrow \mathbb{I}^{2}$ for the full subcategory that is the pair groupoid on the boundary of $I^{2}$.

When we work with arbitrary groupoids in a well-adapted model $\mathcal{E}$ of synthetic differential geometry it is necessary to work with epimorphisms between objects of $\mathcal{E}$ than between their sets of global sections. Hence we make the following definitions:

Definition 1.8. A groupoid $\mathbb{G}$ in $\mathcal{E}$ is $\mathcal{E}$-path connected iff

$$
\mathbb{G}^{\mathbb{I}} \stackrel{\mathbb{G}^{\iota \mathbb{I}}}{\longrightarrow} \mathbb{G}^{\partial \mathbb{I}}
$$

is an epimorphism in $\mathcal{E}$. A groupoid $\mathbb{G}$ in $\mathcal{E}$ is $\mathcal{E}$-simply connected iff it is $\mathcal{E}$-path connected and

$$
\mathbb{G}^{\mathbb{I}^{2}} \stackrel{\mathbb{G}^{\iota} \mathbb{I}^{2}}{\longrightarrow} \mathbb{G}^{\partial \mathbb{I}^{2}}
$$

is an epimorphism in $\mathcal{E}$.

This means that for an arbitrary groupoid in $\mathcal{E}$ being $\mathcal{E}$-connected is a stronger condition to impose than being source connected. In Section 2 we show that a Hausdorff Lie groupoid is source path/simply connected iff it is $\mathcal{E}$-path/E-simply connected.

\subsection{The jet part}

The linear approximation of a Lie groupoid has the structure of a Lie algebroid (see for instance Section 3.5 of [21]). By contrast in [5] we define an analytic approximation of an arbitrary groupoid in $\mathcal{E}$. This new structure approximates a Lie groupoid in an analogous way to how a formal group law approximates a Lie group. In this section we briefly sketch the main features of this analytic approximation.

Using the infinitesimal objects of synthetic differential geometry we can define an infinitesimal neighbour relation $\sim$. Intuitively speaking $a \sim b$ expresses that $b$ is contained in an infinitesimal jet based at $a$. For more details see Section 3.1. Using this neighbour relation we can define the jet part $\mathbb{G}_{\infty}$ of a groupoid $\mathbb{G}$ with object space $G$ and arrow space $M$ that consists of all the arrows that are infinitely close to an identity arrow. In [5] we show that this jet part is closed under composition and so defines a subcategory

$$
\mathbb{G}_{\infty} \stackrel{\iota_{\mathbb{G}}^{\infty}}{\longrightarrow} \mathbb{G}
$$

which is however not in general a groupoid.

\subsubsection{Symmetry of the neighbour relation}

It turns out that the neighbour relation $\sim$ is not symmetric for all objects of $\mathcal{E}$. In fact it is not symmetric on the object $D$ of all nilsquares in the real line. In [5] we show that this implies that the jet part $\nabla D_{\infty}$ of the pair groupoid $\nabla D$ on $D$ is not a groupoid (although it is a category). Fortunately in [5] we also show that the symmetry of $\sim$ in the arrow space of a groupoid $\mathbb{G}$ is not only a necessary condition but also a sufficient condition to ensure that the jet part $\mathbb{G}_{\infty}$ of $\mathbb{G}$ is a groupoid. We justify this assumption in Section 3.3 by showing that the neighbour relation is symmetric for all classical Hausdorff Lie groupoids. 


\subsubsection{Path connectedness of the jet part}

When we prove Lie's second theorem in [5] there is an additional condition that is required which is not visible in the classical theory. In addition to requiring that a groupoid satisfies certain connectedness and completeness conditions we need to assume that its jet part is $\mathcal{E}$-path connected. We justify this assumption in Section 3.6 by showing that the jet part of every classical Hausdorff Lie groupoid is $\mathcal{E}$-path connected.

\subsection{Integral completeness}

To construct global data from local data in classical Lie theory we use the fact that all smooth vector fields admit a unique local solution when we fix an initial vector. Unfortunately when we replace the category Man with a well-adapted model $\mathcal{E}$ of synthetic differential geometry we can no longer use this result. In this section we identify a class of groupoids for which we can construct global data from local data. It turns out that we do not need to assert the existence of all solutions to smooth vector fields but instead a weaker condition suffices. In [6] we see that the crucial lifting property required to prove Lie's second theorem involves the integration of a certain type of path in a Lie algebroid (called $A$-paths) to a certain type of path in a Lie groupoid (called $G$-paths).

Let $\mathbb{I}$ be the pair groupoid on the unit interval $I$ and $\mathbb{G}$ be a Lie groupoid with arrow space $G$ and object space $M$. In Section 4.3 we show that $A$-paths correspond to global sections of the object $\mathbb{G}^{\mathbb{I}} \infty$ in $\mathcal{E}$ and $G$-paths correspond to global sections of the object $\mathbb{G}^{\mathbb{I}}$. Hence we restrict attention to groupoids that are integral complete in the following sense:

Definition 1.9. A groupoid $\mathbb{G}$ in $\mathcal{E}$ is integral complete iff

$$
\mathbb{G}^{\mathbb{I}} \stackrel{\mathbb{G}^{I_{1}}}{\longrightarrow} \mathbb{G}^{\mathbb{I}_{\infty}}
$$

is an isomorphism in $\operatorname{Gpd}(\mathcal{E})$.

This assumption is a crucial one in the proof of Lie's second theorem presented in [5] and so in Section 4 we justify it by proving that all classical Hausdorff Lie groupoids are integral complete.

\section{Path and simply connectedness}

In this section we show that for all Hausdorff Lie groupoids $\mathbb{G}$ with arrow space $G$ and object space $M$ the classical source path and source simply connectedness conditions coincide with their internal counterparts. (Please see Section 1.3 for the relevant definitions.) In other words, we show that if $\mathbb{G}$ is source path connected then $\mathbb{G}$ is $\mathcal{E}$-path connected and if further $\mathbb{G}$ is source simply connected then $\mathbb{G}$ is $\mathcal{E}$-simply connected. To do this we will need an explicit description of the coverage that generates the well-adapted model $\mathcal{E}$. Hence for this section we will assume that $\mathcal{E}$ is the Dubuc topos which is generated by the Dubuc site as defined in Section 1.2. Note that this means that our results hold for all the well-adapted models generated by a site contained in the Dubuc site. For instance by referring to Appendix 2 of [23] we see that our results hold for the Cahiers topos (see [9]) and the classifying topos of local Archimedean $C^{\infty}$-rings (see Appendix 2 of [23]).

We deduce both the path connected and simply connected results from the following stronger result.

Notation 2.1. Let $B$ be a compact and contractible subset of a Euclidean space that is a zero set of an ideal of smooth functions $I$ :

$$
B=[n, I]=\left\{\vec{x} \in \mathbb{R}^{n}: \forall \phi \in I, \phi(\vec{x})=0\right\},
$$


which means that we can view $B$ as a representable object in the Dubuc topos as well as a subset of Euclidean space.

Let $\partial B$ denote the boundary of $B$ and $\nabla B$ and $\nabla \partial B$ be the pair groupoids on $B$ and $\partial B$ respectively. (Recall that the pair groupoid has precisely one invertible arrow between any pair of objects.) There is a natural inclusion $\iota_{B}: \nabla \partial B \rightarrow \nabla B$.

Notation 2.2. We write $r \in_{X} R$ to denote that $r$ is an arrow $X \rightarrow R$ in $\mathcal{E}$ and say that $r$ is a generalised element of $R$ at stage of definition $X$.

We prove that if every global element $f \in \in_{1} \mathbb{G}^{\nabla \partial B}$ has a filler $F \in_{1} \mathbb{G}^{\nabla B}$ (i.e., $G^{\iota_{B}} F=f$ ) then the arrow

$$
\mathbb{G}^{\iota_{B}}: \mathbb{G}^{\nabla B} \rightarrow \mathbb{G}^{\nabla \partial B}
$$

is an epimorphism in $\mathcal{E}$. Note that being $\mathcal{E}$-path connected is the case when $B$ is the unit interval $I$ and being $\mathcal{E}$-simply connected is the conjunction of the cases $B=I$ and $B=I^{2}$.

Our general strategy will be to split the tangent bundle using the submersion $s$ and then show that various constructions involving Riemannian exponential maps can be forced to respect this splitting. Once this is done we can work in just one source fibre where the result is substantially easier.

However first we need to consider the interrelationships between various kinds of open subset and subobject possible in the context of a well-adapted model of synthetic differential geometry.

\subsection{Open subobjects of function spaces}

Our aim is to show that a certain arrow between function spaces is an epimorphism. As is the case for all Grothendieck toposes, the epimorphisms in the Dubuc topos are characterised in terms of the coverage that is used to generate the topos. However the natural and convenient notion of open subset for a space of smooth functions is the smooth compact-open topology. In order to mediate between these two notions of open subobject/subset we introduce another type of open subobject due to Penon. First we recall that every Penon open subobject of a representable object is a Dubuc open subobject. Then we recall that any smooth compactopen subset of the set of global sections of a function space induces a Penon open subobject of the function space.

\subsubsection{Penon open subobjects}

In this section we will briefly sketch some of the theory of topological structures in synthetic differential geometry and refer to [3] and [25] for more comprehensive accounts. Following Penon in [24] we say that an element $r$ of the line object $R$ in the Dubuc topos is infinitesimal iff

$$
\neg \neg(r=0)
$$

holds in the internal logic of the Dubuc topos. Since the line object contains nilpotent elements it is not a field. However it is easy to see that all of these nilpotents are infinitesimal as defined above and in fact Theorem 10.1 in [17] tells us that the line object $R$ is a field of fractions, which is to say that for all elements $r \in R$ the proposition

$$
\neg(r=0) \Longleftrightarrow(r \text { is invertible })
$$

holds. We note that in the context of classical logic being a field of fractions implies that every element is either zero or invertible but this implication does not hold for intuitionistic 
logic. Using the correspondence in (2.1) we can deduce that the infinitesimals and the invertible elements of the line object are separated in the following sense: for all $r, s \in R$ the proposition

$$
\neg(r=0) \wedge \neg \neg(s=0) \Longrightarrow \neg(r=s)
$$

holds. The following definition is Definition 1.5 in [11].

Definition 2.3. A subobject $U \subset X$ in $\mathcal{E}$ is Penon open iff the proposition

$$
\forall u \in U, \quad \forall x \in X, \quad(x \in U) \vee(\neg(u=x))
$$

holds in the internal logic of $\mathcal{E}$.

Remark 2.4. If $U$ is Penon open then for all $u \in U$ then there is an inclusion

$$
\{x: \neg \neg(x=u)\} \subset U \text {. }
$$

Example 2.5. The subobject

$$
\{r \in R: \neg(r=0)\}
$$

of $R$ is a Penon open subset.

In fact we can give a characterisation of the Penon open subsets of representable objects in terms of classical open subsets. Recall from [10] and Lemma 1.3 in III.1 of [23] that the site generating the Dubuc topos is subcanonical and that by construction the image of any open subset under the full and faithful embedding of the category of smooth manifolds into the Dubuc topos is a Dubuc open subobject. The following is Corollary 8 in [12].

Proposition 2.6. A subobject $U$ of a representable object $y X$ in the Dubuc topos is Penon open iff it is of the form $U=\iota V \cap y X$ for some open subset $V \subset \mathbb{R}^{n}$.

Corollary 2.7. If $X$ is a Penon open subobject of a representable then it is Dubuc open.

Finally we record the result that arbitrary Penon open subobjects are stable under pullback.

Corollary 2.8. Let $f: W \rightarrow X$ be an arrow and $U$ be a Penon open subobject of $X$. Then

$$
f^{*} U=\{w \in W: f w \in U\}
$$

is a Penon open subobject of $W$.

Proof. The hypothesis that $U$ is a Penon open subobject of $X$ implies that for all $w \in W$ and $v \in f^{*} U$ the proposition

$$
(f w \in U) \vee(\neg(f w=f v))
$$

holds. But by definition $f w \in U$ iff $w \in f^{*} U$ and it is immediate that $\neg(f w=f v)$ implies that $\neg(w=v)$. 


\subsubsection{Smooth compact-open subsets}

Recall that for any topos $\mathcal{E}$ the global sections functor $\Gamma$ restricts to a functor from the poset of subobjects of some function space $Y^{X}$ to the poset of subsets of $\Gamma\left(Y^{X}\right)$. In fact when $\mathcal{E}$ is the Dubuc topos $\Gamma$ has a right adjoint

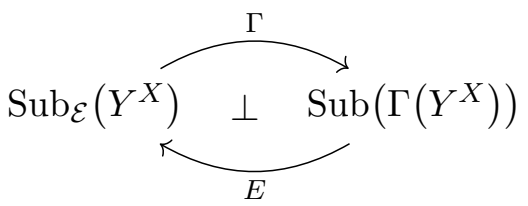

for which the unit is an isomorphism (see Lemma 1.5 in [2]). Therefore it is natural to ask whether we can characterise the subsets $U$ of $\Gamma\left(Y^{X}\right)$ for which $E U$ is a Penon open subobject. Let $M, N$ be smooth manifolds and $f: M \rightarrow N$ a smooth function. Let $(\phi, V)$ and $(\psi, W)$ be charts for $M$ and $N$ respectively and let $K$ be a compact subset of $V$ such that $f(K) \subset W$. Then following Section 2.1 in [15] we define the weak subbasic neighbourhood $U_{f, \epsilon, K}^{\phi, \psi}$ of $C^{\infty}(M, N)$ to be the set of all smooth functions $g: M \rightarrow N$ such that $g(K) \subset V$ and such that for all integers $k$ the inequality

$$
\left|D^{k}\left(\psi f \phi^{-1}\right)(m)-D^{k}\left(\psi g \phi^{-1}\right)(m)\right|<\epsilon
$$

holds for all $m \in \phi(K)$. We call the topology generated by the weak subbasic neighbourhoods the smooth compact-open topology. The following is Proposition 1.6 of [2].

Proposition 2.9. If $U$ is a smooth compact-open subset of $\Gamma\left(Y^{X}\right)$ then $E U$ is a Penon open subobject.

Remark 2.10. If $f \in U$ then $f: 1 \rightarrow Y^{X}$ factors through $E U \mapsto Y^{X}$.

\subsection{Splitting the tangent bundle}

In this section we use the source submersion $s: G \rightarrow M$ of a Lie groupoid $G \rightrightarrows M$ to split the tangent bundle $T G \rightarrow G$ into horizontal and vertical components. We then confirm that when we pullback the tangent bundle to a contractible base space this splitting is maintained in the trivial bundle that results.

Definition 2.11. If $p_{H}: H \rightarrow G$ and $p_{V}: V \rightarrow G$ are vector bundles then the direct sum $H \oplus V$ is defined as the pullback vector bundle

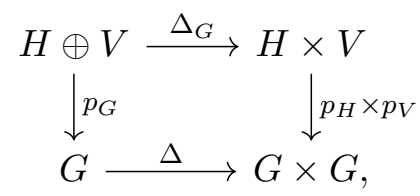

where $\Delta$ is the diagonal.

Notation 2.12. Let $\mathbb{G}$ be a Lie groupoid with arrows space $G$, object space $M$, source map $s$ and a Riemannian metric $\sigma^{G}$ on $G$. Let $B$ be a contractible subset of a Euclidean space $\mathbb{R}^{n}$ that is the zero set of an ideal of smooth functions. Let $p_{V}: V=\operatorname{ker}\left(s^{D}\right) \rightarrow G$ and $p_{H}: H=\left(\operatorname{ker}\left(s^{D}\right)\right)^{\perp}$ $\rightarrow G$ be vector bundles where the orthogonal complement is defined using the metric $\sigma$.

First we note that the squares
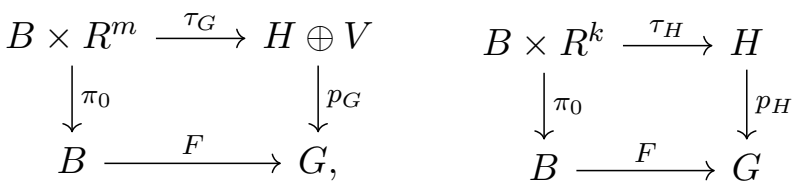

and

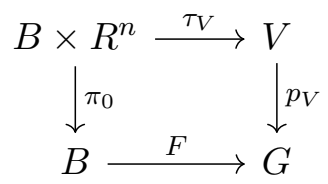


are pullbacks for some natural numbers $m, k$ and $n$ because $B$ is contractible. By construction $T G \cong H \oplus V$ as vector bundles.

Lemma 2.13. The square

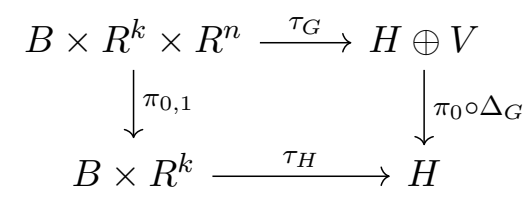

commutes.

Proof. There is a unique $\psi: B \times R^{k} \times R^{n} \rightarrow H \oplus V$ making

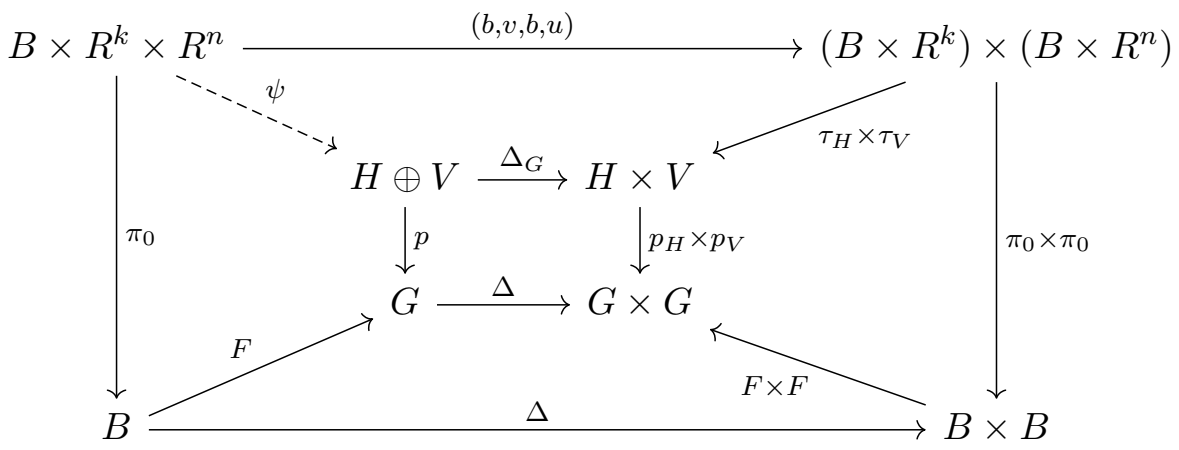

into a commutative cube because the centre square is a pullback. Furthermore the right and outer squares are easily seen to be pullbacks. This means that the left square is a pullback and $\psi=\tau_{G}$. Now the result follows from the fact that

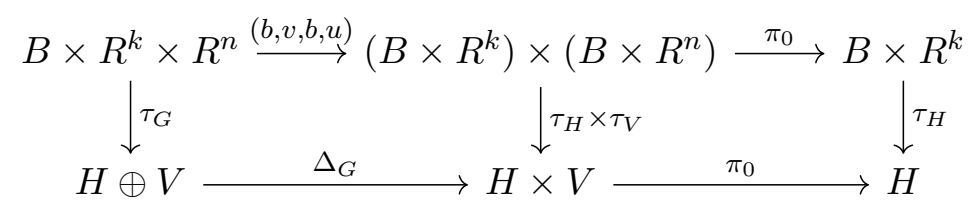

commutes.

Corollary 2.14. There is an isomorphism $\alpha$ making

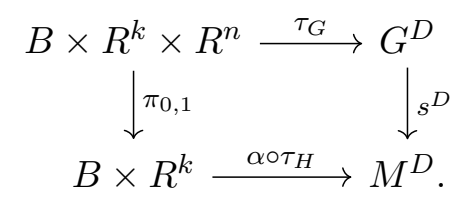

Proof. Since $s^{D}$ is an epimorphism there is an isomorphism $\alpha$ making

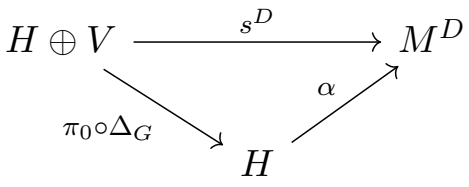

commute. Then result follows immediately from Lemma 2.13. 


\subsection{Riemannian submersions}

In order to transfer fillers between neighbouring source fibres we need to know how to transport them in parallel to the source fibres. To do this we will use the exponential map on the arrow space $G$ induced by a Riemannian metric on $G$. However it is not in general true that for arbitrary Riemannian metrics $\eta^{G}$ and $\eta^{M}$ on $G$ and $M$ respectively that $s$ maps geodesics with respect to $\eta^{G}$ to geodesics with respect to $\eta^{M}$.

We now recall a little of the theory of Riemannian submersions which will allow us to construct Riemannian metrics $\sigma^{G}$ on $G$ and $\sigma^{M}$ on $M$ such that $s$ maps geodesics with respect to $\sigma^{G}$ to geodesics with respect to $\sigma^{M}$.

Notation 2.15. Let $G$ and $M$ be smooth manifolds with Riemannian metrics $\eta^{G}$ and $\eta^{M}$ respectively. Let $s: G \rightarrow M$ be a submersion. We write $\operatorname{ker}(s)=V$ for the sub-bundle of the tangent bundle $G^{D}$ that is parallel to the $s$-fibres and $H=(\operatorname{ker}(s))^{\perp}$ for the bundle orthogonal to $V$ with respect to the Riemannian metric $\eta^{G}$. Let $U^{G}$ and $U^{M}$ denote the domains of the exponential maps associated to $\eta^{G}$ and $\eta^{M}$ respectively.

The next definition is part of the Definition 26.9 in [22].

Definition 2.16. The submersion $s$ is a Riemannian submersion iff

$$
\left(\left.s^{D}\right|_{H}\right)_{g}: H_{g} \rightarrow\left(M^{D}\right)_{s(g)}
$$

is an isometric isomorphism.

The next result is Lemma 2.1.1 in [7].

Lemma 2.17. If $s: G \rightarrow M$ is a submersion then we can choose Riemannian metrics $\sigma^{G}$ and $\sigma^{M}$ on $G$ and $M$ respectively that make $s$ a Riemannian submersion.

Proof. To begin with choose arbitrary Riemannian metrics $\eta^{G}$ and $\eta^{M}$ on $G$ and $M$ respectively. Use $\eta^{G}$ to decompose $G^{D}=(\operatorname{ker}(s))^{\perp} \oplus \operatorname{ker}(s)=H \oplus V$. Now we can define an alternative positive definite inner product $\sigma^{H}$ on $H$ as the pullback of $\eta^{M}$ along the isometry $\left(\left.s^{D}\right|_{H}\right)$. Also we can restrict the Riemannian metric $\eta^{G}$ to a positive definite inner products $\sigma^{V}$ on $V$. Then we define a new Riemannian metric $\sigma^{G}$ on $G$ by declaring all vectors in $H$ to be orthogonal to all vectors in $V$. By construction $s$ is a Riemannian submersion with respect to $\sigma^{G}$ and $\eta^{M}$.

Lemma 2.18. If $s: G \rightarrow M$ is a Riemannian submersion and $c:[0,1] \rightarrow G$ is a geodesic in $G$ such that $c^{\prime}(0) \in H$ then $s \circ c$ is a geodesic in $M$. Furthermore if $a \in[0,1]$ then $c^{\prime}(a) \in H$.

Proof. This is Corollary 26.12 in [22].

Corollary 2.19. If $s: G \rightarrow M$ is a Riemannian submersion then there exist open subsets $W^{M} \subset U^{M}$ and $W^{G} \subset U^{G}$ such that

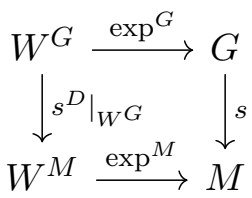

commutes.

Proof. Immediate from Lemma 2.18. See also Proposition 5.9 in [7]. 


\subsection{Constructing a tubular extension}

In this section we construct a tubular extension $B \times C^{k} \times C^{n} \rightarrow G$ for every smooth map $F: B \rightarrow G$ where $G$ is the arrow space of a Hausdorff Lie groupoid. In the next two sections we work within this tubular extension to construct the Penon open subobject that we need. We also show that this extension commutes in the appropriate way with the source map.

Notation 2.20. Let $C^{n}$ denote the open unit hypercube in $R^{n}$. Let $B$ be a contractible and compact subset of Euclidean space that is the zero set of an ideal of smooth functions.

Lemma 2.21. If $\bar{W}^{G}=W^{G} \cap\left(s^{D}\right)^{-1}\left(W^{M}\right)$ then there are open inclusions $\nu^{G}: C^{k} \rightarrow R^{k}$ and $\nu^{M}: C^{n} \rightarrow R^{n}$ such that $\nu^{G}(\overrightarrow{0})=\overrightarrow{0}$ and $\nu^{M}(\overrightarrow{0})=\overrightarrow{0}$ and maps $\iota^{G}: B \times C^{k} \times C^{n} \rightarrow \bar{W}^{G}$ and $\iota^{M}: B \times C^{k} \rightarrow W^{M}$ such that

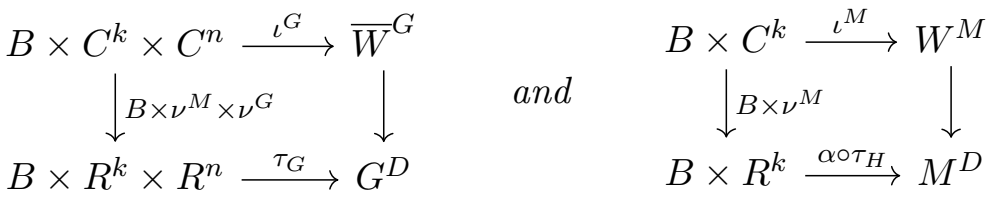

commute.

Proof. By construction for each $b \in B$ the arrow $\tau_{G}(b,-,-): R^{k} \times R^{n} \rightarrow G_{b}^{D}$ is an isomorphism. Therefore $X_{b}=\tau_{G}(b,-,-)^{-1}\left(\bar{W}_{b}^{G}\right)$ specifies a collection of open sets containing $\overrightarrow{0}$ in $R^{k+n}$ that vary smoothly with $B$. Since $B$ is compact we can find an open ball around $\overrightarrow{0}$ contained in each of the $X_{b}$. Now the existence of $\iota^{G}$ and $\nu^{G}$ follows easily. The existence of $\iota^{M}$ and $\nu^{M}$ follows similarly.

Lemma 2.22. If $F: B \rightarrow G$ is a smooth map that is s-constant and starts at an identity arrow then there exist smooth maps $\xi^{G}: B \times C^{k} \times C^{n} \rightarrow G$ and $\xi^{M}: B \times C^{k} \rightarrow M$ such that

- both $\xi^{G}(b, 0,0)=F(b)$ and $\xi^{N}(b, 0,0)=F(b)$,

- for all $b \in B$ both $\xi^{G}(b,-,-)$ and $\xi^{M}(b,-,-)$ are open inclusions,

- the diagram

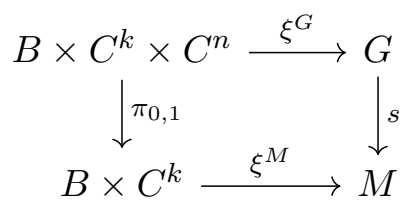

commutes.

Proof. If $\bar{W}^{G}=W^{G} \cap\left(s^{D}\right)^{-1}\left(W^{M}\right)$ then in the cube

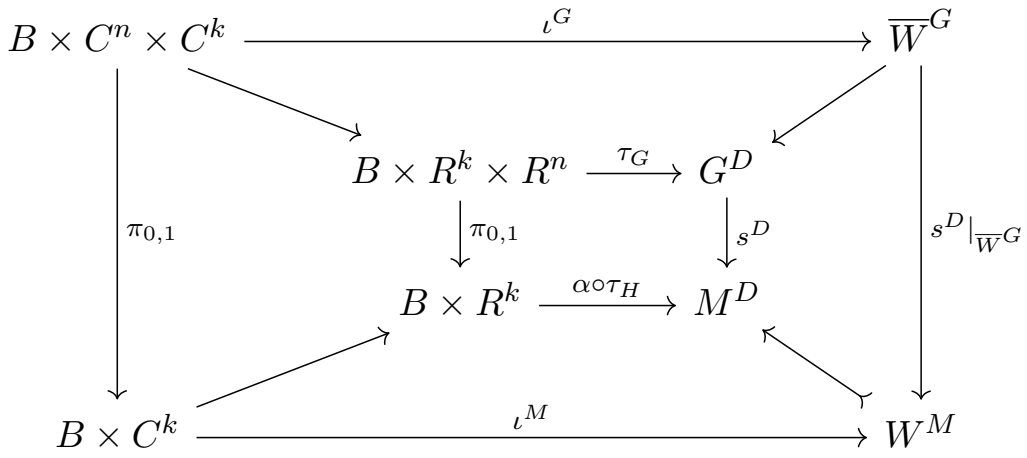


the centre square commutes by Corollary 2.14, the upper and lower squares commute by Lemma 2.21 and the left and right squares commute by construction. Therefore the outer square commutes because $W^{M} \longmapsto M^{D}$ is a monomorphism. The result now follows from pasting the square shown to commute in Corollary 2.19 onto the right of the above square; the maps we require are $\xi^{G}=\exp ^{G} \circ \iota^{G}$ and $\xi^{M}=\exp ^{M} \circ \iota^{M}$.

\subsection{A subobject of the tubular extension admitting fillers}

In the previous section we constructed a tubular extension $\xi_{F}^{G}: B \times C^{k} \times C^{n} \rightarrow G$ for every $F \in \mathbb{G}^{\nabla B}$. In this section we construct a subobject of $\mathbb{G}^{\nabla \partial B}$ from this tubular extension such that every element of this subobject admits a filler. In the next section we find a Penon open subobject contained in this subobject.

Notation 2.23. Let $B$ be a subset of Euclidean space that is the zero set of an ideal of smooth functions. Let $f \in \mathbb{G}^{\nabla \partial B}$ have a filler $F \in \mathbb{G}^{\nabla B}$. We write $\xi_{F}^{G}$ for the tubular extension constructed in Section 2.4.

Remark 2.24. For all $\vec{x}_{0} \in C^{k}$ the map $\partial B \rightarrow G$ defined by $b \mapsto \xi_{F}^{G}\left(b, \vec{x}_{0}, \overrightarrow{0}\right)$ has filler $B \rightarrow G$ defined by $b \mapsto \xi_{F}^{G}\left(b, \vec{x}_{0}, \overrightarrow{0}\right)$.

Definition 2.25. The subobject $T_{f} \longmapsto \mathbb{G}^{\nabla \partial B}$ consists of all $\chi \in \mathbb{G}^{\nabla \partial B}$ such that

$$
\forall b \in B, \quad \chi(b) \in \xi_{F}^{G}\left(b, C^{k}, C^{n}\right)
$$

or equivalently $T_{f}$ is the subobject of $\mathbb{G}^{\nabla \partial B}$ such that

$$
\forall b \in B, \quad \exists \vec{x}_{0} \in C^{k}, \quad \exists h_{\chi} \in\left(C^{n}\right)^{\partial B}, \quad \chi(b)=\xi_{F}^{G}\left(b, \vec{x}_{0}, h_{\chi}(b)\right),
$$

because $\chi$ is source constant.

Remark 2.26. By construction $F(b)=\xi_{F}^{G}(b, \overrightarrow{0}, \overrightarrow{0})$. Restricting to $\partial B$ gives that $f(b)=$ $\xi_{F}^{G}(b, \overrightarrow{0}, \overrightarrow{0})$ and hence $f \in T_{f}$.

Lemma 2.27. If $\chi \in T_{f}$ then $\chi$ has a filler $X \in \mathbb{G}^{\nabla B}$.

Proof. If $\chi(b)=\xi_{F}^{G}\left(b, \vec{x}_{0}, h_{\chi}(b)\right)$ then there is an homotopy from $\chi$ to $\left(b \mapsto \xi_{F}^{G}\left(b, \vec{x}_{0}, \overrightarrow{0}\right)\right)$ defined by

$$
\begin{aligned}
I \times \partial B & \rightarrow G, \\
(a, b) & \mapsto \xi_{f}^{G}\left(b, \vec{x}_{0},(1-a) h_{\chi}(b)\right),
\end{aligned}
$$

and composing this homotopy with the filler $\left(b \mapsto \xi_{F}^{G}\left(b, \vec{x}_{0}, \overrightarrow{0}\right)\right)$ is a filler for $\chi$.

\subsection{A compact-open set inside a tubular extension}

In this section we identify a compact-open set that is contained in space of global sections of $\mathbb{G}^{\nabla \partial B}$ that is contained in the subobject $T_{f}$ constructed in Section 2.5. Once we have done this we can deduce using Proposition 2.9 the existence of a Penon open subobject $V_{f}$ of $\mathbb{G}^{\nabla \partial B}$ such that all maps in $V_{f}$ have fillers.

Notation 2.28. Let $B$ be a subset of Euclidean space that is the zero set of an ideal of smooth functions. Let $f \in \mathbb{G}^{\nabla \partial B}$ have a filler $F \in \mathbb{G}^{\nabla B}$. We write $\xi_{F}^{G}$ for the tubular extension constructed in Section 2.4. Let $D^{n} \longmapsto C^{n}$ be the inclusion of the ball of radius $\frac{1}{2}$ centred at the origin. Let $E^{n} \longmapsto D^{n}$ be the inclusion of the ball of radius $\frac{1}{4}$ centred at the origin, 
Definition 2.29. The compact-open subset $W_{f}$ of $\Gamma\left(\mathbb{G}^{\nabla \partial B}\right)$ is defined as follows. Let $U_{b}=$ $f^{-1} \xi_{F}^{G}\left(b, E^{k}, E^{n}\right)$. Now $\left(U_{b}\right)_{b \in \partial B}$ covers $\partial B$ because $b \in U_{b}$. Since $\partial B$ is compact we can choose $b_{1}, \ldots, b_{n} \in \partial B$ such that $\left(U_{b_{i}}\right)_{i=1}^{n}$ covers $\partial B$. The compact-open set $W_{f}$ that we require is defined by the family $\left(\overline{U_{b_{i}}}, \xi_{F}^{G}\left(b_{i}, D^{k}, D^{n}\right)\right)_{i=1}^{n}$.

Remark 2.30. Note that $f \in W_{f}$ because $f\left(U_{b}\right)=\xi_{F}^{G}\left(b, E^{k}, E^{n}\right)$ and so $f\left(\overline{U_{b}}\right) \subset \xi_{F}^{G}\left(b, D^{k}, D^{n}\right)$.

Lemma 2.31. The compact-open set $W_{f}$ of $\Gamma\left(\mathbb{G}^{\nabla \partial B}\right)$ is contained in $\Gamma\left(T_{f}\right)$.

Proof. Let $\chi \in W_{f}$. For each $b \in \partial B$ there exists at least one $i \in\{1, \ldots, n\}$ such that $b \in U_{b_{i}}$. For all such $i$ the elements $f(b)$ and $\chi(b)$ are in the open set $\xi_{F}^{G}\left(b_{i}, D^{k}, D^{n}\right)$ of $G$. Now for each $b \in \partial B$ the map $\iota^{G}(b,-,-)$ preserves distances. Furthermore since $\xi_{F}^{G}=\exp ^{G} \iota_{\iota} G$ the map $\xi_{F}^{G}(b,-,-)$ preserves distances from the origin. Finally recall that $f\left(b_{i}\right)=\xi_{F}^{G}\left(b_{i}, \overrightarrow{0}, \overrightarrow{0}\right)$. Hence

$$
d(\chi(b), f(b)) \leq d\left(\chi(b), f\left(b_{i}\right)\right)+d\left(f\left(b_{i}\right), f(b)\right)<\frac{1}{2}+\frac{1}{2}=1,
$$

which tells us that in fact $\chi \in \Gamma\left(T_{f}\right)$. So $W_{f} \subset \Gamma\left(T_{f}\right)$.

Corollary 2.32. If $f \in \mathbb{G}^{\nabla \partial B}$ has filler $F \in \mathbb{G}^{\nabla B}$ then there exists a Penon open subobject $\Phi: V_{f} \longmapsto \mathbb{G}^{\nabla \partial B}$ and a lift $\Psi: V_{f} \rightarrow \mathbb{G}^{\nabla B}$ making

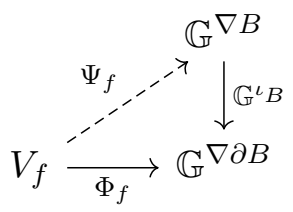

commute.

Proof. Let $V_{f}=E\left(W_{f}\right)$ where $E$ is the left adjoint to the global sections functor as in Section 2.1.2. Note that by construction $f \in \Gamma\left(V_{f}\right)$.

\subsection{Ordinary connectedness implies internal connectedness}

Now we are in a position to deduce the main result of this paper. Let $\mathbb{G}$ be a (Hausdorff) Lie groupoid with arrow space $G$ and object space $M$.

Theorem 2.33. If $B$ is a compact and contractible subset of Euclidean space that is the zero set of an ideal of smooth functions then the arrow $\mathbb{G}^{\iota_{B}}: \mathbb{G}^{\nabla B} \rightarrow \mathbb{G}^{\nabla \partial B}$ is an epimorphism.

Proof. We perform a sequence of reductions to show that it in fact suffices to prove Corollary 2.32.

Firstly, to show that $\mathbb{G}^{\iota B}$ is an epimorphism, it will suffice to show that for all representable objects $X$ in $\mathcal{E}$ and arrows $\phi: X \rightarrow \mathbb{G}^{\nabla \partial B}$ in $\mathcal{E}$ there exists a Dubuc open cover $\left(\iota_{i}: X_{i} \rightarrow X\right)_{i \in I}$ such that for all $i \in I$ there exists a lift $\psi_{i}$ making

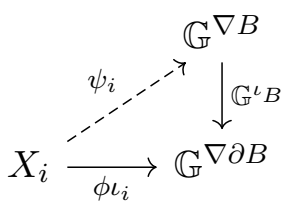

commute. 
In fact it will suffice to find for each $f \in_{1} \mathbb{G}^{\nabla \partial B}$ a Penon open subobject $U_{f}$ of $\mathbb{G}^{\nabla \partial B}$ containing $f$ and a lift

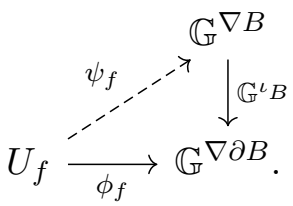

Indeed $\left(U_{f}\right)_{f \in \mathbb{G} \nabla \partial B}$ covers $\mathbb{G}^{\nabla \partial B}$ as Penon open subobjects and so the pullback cover $\left(\phi^{-1}\left(U_{f}\right)\right)_{f \in \mathbb{G} \nabla \partial B}$ covers $X$ as Penon open subobject. But now we use Corollary 2.7 and the fact that $X$ is representable to see that $\left(U_{f}\right)_{f \in \mathbb{G} \nabla \partial B}$ covers $\mathbb{G}^{\nabla \partial B}$ as Dubuc open subobjects also.

But the existence of $\psi_{f}$ and a Penon open $\phi_{f}$ making (2.2) commute is the conclusion of Corollary 2.32.

Corollary 2.34. If $\mathbb{G}$ is an s-path connected Lie groupoid then the arrow $\mathbb{G}^{\iota_{I}}: \mathbb{G}^{\nabla I} \rightarrow \mathbb{G}^{2}$ is an epimorphism and so, by definition, the groupoid $\mathbb{G}$ is internally path connected.

Corollary 2.35. If $\mathbb{G}$ is an s-simply connected Lie groupoid then the arrow $\mathbb{G}^{\iota(I \times I)}: \mathbb{G}^{\nabla(I \times I)} \rightarrow$ $\mathbb{G}^{\nabla \partial(I \times I)}$ is an epimorphism and so, by definition, the groupoid $\mathbb{G}$ is internally simply connected.

\section{Properties of the jet part}

\subsection{The infinitesimal neighbour relation}

In this section we introduce the infinitesimal neighbour relation which is used to define the jet part of a category in [5]. If $\mathbb{C}$ is a category in any well-adapted model $\mathcal{E}$ of synthetic differential geometry and $M$ is the space of objects of $\mathbb{C}$ then we define the infinitesimal neighbour relation on objects of the slice topos $\mathcal{E} / M$. In [5] we justify this choice by showing that the jet part defined using this neighbour relation is closed under composition in $\mathbb{C}$.

Let $a, b: X \rightarrow B$ where $X$ and $B$ are objects of the topos $\mathcal{E} / M$. Then $a \sim b$ iff there exists a cover $\left(\iota_{i}: X_{i} \rightarrow X\right)_{i \in I}$ in $\mathcal{E} / M$ such that for each $i$ there exists an object $D_{W_{i}} \in \operatorname{Spec}($ Weil), an arrow $\phi_{i}: X_{i} \times D_{W_{i}} \rightarrow B$ and an arrow $d_{i}: X_{i} \rightarrow D_{W_{i}}$ such that

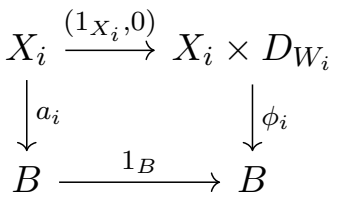

and

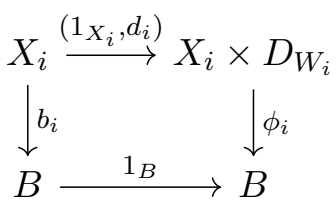

commute, where we have written $a_{i}$ and $b_{i}$ for the restrictions of $a$ and $b$ to $X_{i}$.

Remark 3.1. The relation $\sim$ is not always symmetric. In fact in [5] we see that $\sim$ is not symmetric in the case $B=D$ and $M=1$.

The relation $\approx$ is the transitive closure of $\sim$ in the internal logic of $\mathcal{E} / M$. This means that for $a, b: X \rightarrow B$ we have $a \approx b$ iff there exists a cover $\left(\iota_{i}: X_{i} \rightarrow X\right)_{i \in I}$ and for each $i$ there exists a natural number $n_{i}$ and elements $x_{i_{0}}, x_{i_{1}}, \ldots, x_{i_{n_{i}}} \in X_{X_{i}} B$ such that

$$
a_{i}=x_{i_{0}} \sim x_{i_{1}} \sim \cdots \sim x_{i_{n_{i}}}=b_{i} .
$$




\subsection{The jet factorisation system and the jet part}

In this section we recall the definitions of the jet factorisation system and the jet part of a groupoid.

An arrow $f: A \rightarrow B$ in $\mathcal{E} / M$ is jet-dense iff for all $b: X \rightarrow B$ there exists a cover $\left(\iota_{i}: X_{i} \rightarrow\right.$ $X)_{i \in I}$ and elements $a_{i}: X_{i} \rightarrow A$ such that $f\left(a_{i}\right) \approx b_{i}$. We have written $b_{i}$ for the restriction of $b$ to $X_{i}$. An arrow $g: A \rightarrow B$ in $\mathcal{E} / M$ is jet-closed iff it is a monomorphism and for all $a: X \rightarrow A$ and $b: X \rightarrow B$ such that $g a \approx b$ there exists a cover $\left(\iota_{i}: X_{i} \rightarrow X\right)_{i \in I}$ and elements $c_{i}: X_{i} \rightarrow A$ such that $a_{i} \approx c_{i}$ and $g c_{i}=b_{i}$. We have written $a_{i}$ and $b_{i}$ for the restrictions of $a$ and $b$ respectively to $X_{i}$.

In the case $M=1$ the right class of the jet factorisation system has been studied before. For instance it is the class of formal-etale maps in I.17 of [17]. In fact in Section 1.2 of [16] is it called the class of formally-open morphisms. The sense in which these maps are open is reflected in the following corollary that follows immediately from the definition of jet closed.

Corollary 3.2. The inclusion of an open subset $U$ into a manifold $M$ is jet closed.

Now we recall from [5] the results about the jet factorisation system that we need in the rest of this paper.

Lemma 3.3. Let $h: A \rightarrow E$ be an arrow in $\mathcal{E} / M$. Then there exists a jet closed arrow $g$ and a jet dense arrow $f$ such that $h=g f$. The mediating object in the factorisation has the following description:

$$
B=\{x \in E: \exists a \in A, h a \approx x\} \stackrel{g}{\rightarrow} E .
$$

Proof. See Lemma 3.23 in [5].

Theorem 3.4. Let $\mathbb{G}$ be a groupoid in $\mathcal{E}$. Then the subobject

$$
\left(G_{\infty}, s_{\infty}\right)=\{g \in(G, s): e s g \approx g\}
$$

is closed under composition and hence defines a subgroupoid $\mathbb{G}_{\infty} \longmapsto \mathbb{G}$ called the jet part of $\mathbb{G}$.

Proof. See Corollary 4.4 and Proposition 4.18 in [5].

Proposition 3.5. Let $L_{\infty}$ be the class of jet dense arrows and $R_{\infty}$ the class of jet closed arrows. Then the pair $\left(L_{\infty}, R_{\infty}\right)$ defines a $(\mathcal{E} / M)$-factorisation system.

Proof. See Section 3.2 in [5].

Proposition 3.6. Let $g$ be jet dense and $k$ be jet closed in $\mathcal{E} / M$. Suppose that the relation $\approx$ is symmetric on the object $E$ and that the square

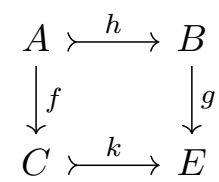

is a pullback. Then $f$ is also jet dense.

Proof. See Proposition 3.27 in [5]. 


\subsection{Neighbour relation is symmetric for Lie groupoids}

One of the assumptions that is required to prove Lie's second theorem in [5] involves the symmetry of the neighbour relation $\sim$ defined in Section 3.1. More precisely, if $\mathbb{G}$ is a groupoid in $\mathcal{E}$ with arrow space $G$ and source map $s$ then we need to assume that $\sim$ is symmetric on the object $(G, s)$ in $\mathcal{E} / M$. In this section we justify this assumption by proving that if $\mathbb{G}$ is a Lie groupoid then the relation $\sim$ is symmetric on the object $(G, s)$ in $\mathcal{E} / M$.

So suppose that $a, b \in_{X}(G, s)$ in $\mathcal{E} / M$ and $a \sim b$. By definition we have a cover $\left(X_{i} \rightarrow X\right)_{i}$ such that for all $i$ there exist $W_{i} \in \operatorname{Spec}\left(\right.$ Weil), $\phi_{i} \in_{X_{i}}(G, s)^{D_{W_{i}}}$ and $d_{i} \in_{X_{i}} D_{W_{i}}$ making

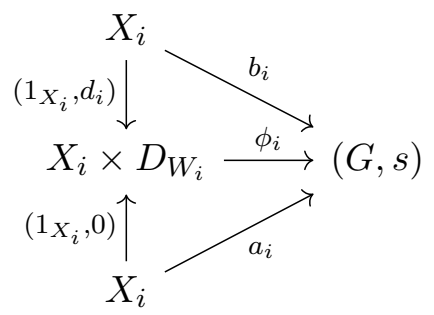

commute where $a_{i}$ and $b_{i}$ are the restrictions of $a$ and $b$ to $X_{i}$. We need to show that $b \sim a$.

Definition 3.7. Let $s: G \rightarrow M$ be an arrow in $M a n$ and $x \in G$. Then a pair of open embeddings $\left(\alpha_{x}, \beta_{x}\right)$ is an $s$-trivialisation centred at $x$ iff

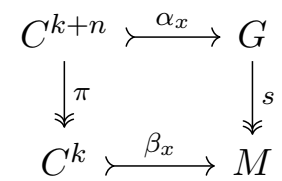

commutes and $\alpha_{x}(0)=x$.

Lemma 3.8. There exists a cover of $\iota_{x}:\left(X_{i, x} \rightarrow X_{i}\right)$ such that $\iota_{x} a_{i}$ factors through an $s$ trivialisation $C^{n+k} \longmapsto G$ around $a_{i}(x)$.

Proof. Let $X_{i}=\left(B_{i}, \xi_{i}\right)$. Since $s$ is a submersion we can choose for each $x \in B_{i}$ an $s$ trivialisation $\nu_{x}: C^{n+k} \longmapsto G$ centred at $a_{i}(x)$. Write $U_{x}$ for the image of $\nu_{x}$. Then the family $\left(\iota_{x}: a_{i}^{-1}\left(U_{x}\right) \rightarrow B_{i}\right)_{x \in B_{i}}$ covers $B_{i}$ in $\mathcal{E}$ and for each $x \in B_{i}$ the arrow $\iota_{x} a_{i}$ factors through $U_{x}$. This means that $\left.\left(\iota_{x}:\left(a_{i}^{-1}\left(U_{x}\right), \xi_{i}\right) \rightarrow X_{i}\right)\right)_{x \in B_{i}}$ is a covering family in $\mathcal{E} / M$ such that $\iota_{x} a_{i}$ factors through $U_{x}$. So we choose $X_{i, x}=\left(\left(a_{i}^{-1}\left(U_{x}\right), \xi_{i}\right)\right.$.

Now using the cover $\left(X_{i, x} \rightarrow X\right)_{i, x}$ we show that $b \sim a$.

Lemma 3.9. If $d_{i, x}, a_{i, x}, b_{i, x}$ and $\phi_{i, x}$ are the restrictions of $d, a, b$ and $\phi$ respectively to $X_{i, x}$ then the arrows $\psi_{i, x}: X_{i, x} \times D_{W_{i}} \rightarrow\left(U_{x}, s\right)$ defined by

$$
\psi_{i, x}(u, d)=a_{i, x}(u)+{ }_{s} \phi_{i, x}\left(u, d_{i, x}(u)\right)-_{s} \phi(u, d)
$$

exhibit $b \sim a$ where $+_{s}$ and $-_{s}$ denote the fibrewise addition and subtraction. (I.e., addition in the last $n$ coordinates of the s-trivialisation.) Hence the infinitesimal neighbourhood relation is symmetric for all Lie groupoids.

Proof. By construction the diagrams

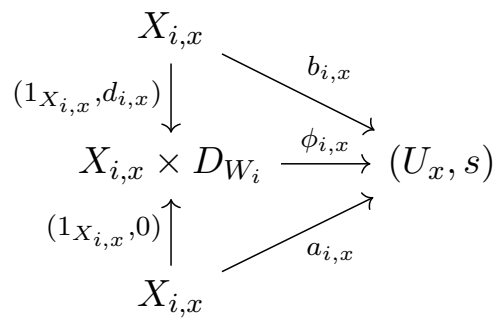


commute for all $x \in B_{i}$. First we check that $\psi_{i, x}$ factors through $U_{x}$. This follows from the equality $\psi(u, 0)=b_{i, x}(u)$ and the fact that the inclusion of $U_{x}$ into $G$ is jet closed. Second we check that $\psi_{i, x}$ defines an arrow in the slice category. But this follows from the fact that the three terms $a_{i, x}(u), \phi_{i, x}\left(u, d_{i, x}(u)\right)$ and $\phi(u, d)$ have the same source and the addition defining $\psi_{i, x}$ is carried out in the last $n$ coordinates of the $s$-trivialisation. Finally since

$$
\psi(u, 0)=a_{i, x}(u)+\phi_{i, x}\left(u, d_{i, x}(u)\right)-\phi_{i, x}(u, 0)=b_{i, x}(u)
$$

and

$$
\psi\left(u, d_{i}(u)\right)=a_{i, x}(u)+\phi_{i, x}\left(u, d_{i, x}(u)\right)-\phi_{i, x}\left(u, d_{i, x}(u)\right)=a_{i, x}(u)
$$

we conclude that $b \sim a$.

\subsection{A trivialisation cover of the identity elements}

In this section we construct a cover $\left(\phi_{e m}: C^{n+k} \rightarrow G\right)_{m \in M}$ of $e(M)$ in $G$ with the property that each $\phi_{e m}$ has a lift $\psi_{e m}$ making

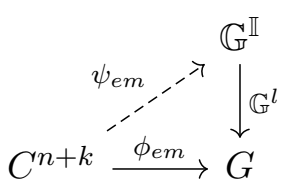

commute and furthermore when we restrict $\psi_{e m}$ to $e(M)$ the fillers we obtain are the constant fillers. First we choose an $s$-trivialisation at $\mathrm{em}$ such that the identity inclusion induces a section of the projection onto the first $k$ coordinates in the trivialisation.

Lemma 3.10. If $m \in M$ then there is an s-trivialisation $\left(\alpha_{e m}, \beta_{\text {em }}\right)$ at em such that e $\beta_{\text {em }}$ factors through $\alpha_{e m}$.

Proof. Let $(\alpha, \beta)$ be any $s$-trivialisation at $\mathrm{em}$. Then if $\nu$ and $\xi$ are defined in the pullback

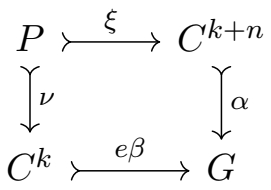

then $\beta \pi \xi=s \alpha \xi=s e \beta \nu=\beta \nu$ and so $\pi \xi=\nu$ because $\beta$ is a monomorphism. Now $P$ is an open set of $C^{k}$ and $0 \in P$ because $e \beta(0)=\alpha(0)$. Since the derivative of $\nu$ has full rank at 0 we can find an open embedding $\iota: C^{k} \longmapsto P$ such that $\nu \iota(0)=0$. Now let $\mu$ be defined by the pullback

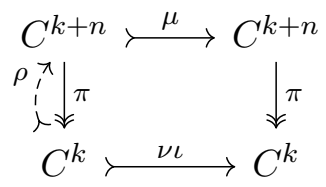

and $\rho$ be induced by the pair $\left(1_{P}, \xi \iota\right)$. Then $e \beta \nu \iota=\alpha \xi \iota=\alpha \mu \rho$ and the $s$-trivialisation that we require is $\left(\alpha_{e m}, \beta_{e m}\right)=(\alpha \mu, \beta \nu \iota)$.

This means that for each $\vec{x} \in C^{k}$ the arrow $\psi(\rho(\vec{x}), \vec{y})$ is an identity arrow. The $\phi_{e m}$ that we require will be the $\alpha_{e m}$ obtained in Lemma 3.10. Now we can construct a lift $\psi_{e m}: C^{k+n} \rightarrow \mathbb{G}^{\mathbb{I}}$ for $\phi_{e m}$ as follows. For each $(\vec{x}, \vec{y}) \in C^{k+n}$ we have a source constant path

$$
a \mapsto(\vec{x}, a \vec{y}+(1-a) \rho(\vec{x})),
$$

which starts at an identity. Since (3.1) is smooth in $\vec{x}$ and $\vec{y}$ it induces an arrow $\psi_{e m}: C^{k+n} \rightarrow \mathbb{G}^{\mathbb{I}}$. Moreover by construction the restriction of $\psi_{e m}$ to $e(M)$ are the constant paths at identity arrows. 


\subsection{A cover of the jet part}

In Section 3.4 we constructed a cover $\left(\phi_{e m}: C^{n+k} \rightarrow G\right)_{m \in M}$ of $e(M)$ in $G$ satisfying certain properties on restriction to $e(M)$. In this section we show that the $\phi_{e m}$ also induce a cover of the object $\left(G_{\infty}, s_{\infty}\right)$ in $\mathcal{E} / M$.

Lemma 3.11. There is an inclusion $j:\left(G_{\infty}, s_{\infty}\right) \longmapsto \bigcup_{m}\left(U_{m}, s \phi_{e m}\right)$ such that $\bigcup_{m} \phi_{e m} \circ j=\iota_{G}^{\infty}$.

Proof. By hypothesis we have an inclusion $\left(M, 1_{M}\right) \longmapsto \bigcup_{m}\left(U_{m}, s \phi_{e m}\right)$ such that $\iota \circ m=e$. Since the inclusion $\iota$ is jet closed in $\mathcal{E} / M$ the square

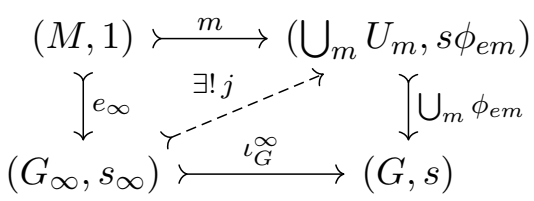

has a unique (monic) filler.

Corollary 3.12. Let the objects $\left(V_{m}, s_{\infty} \phi_{\text {em }}\right)$ of $\mathcal{E} / M$ be defined by the pullbacks

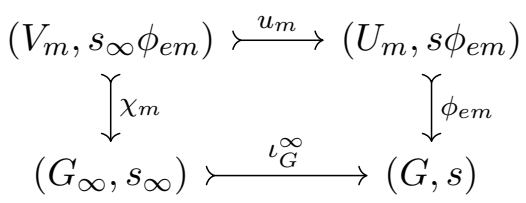

then because colimits are stable under pullback the bottom right square in

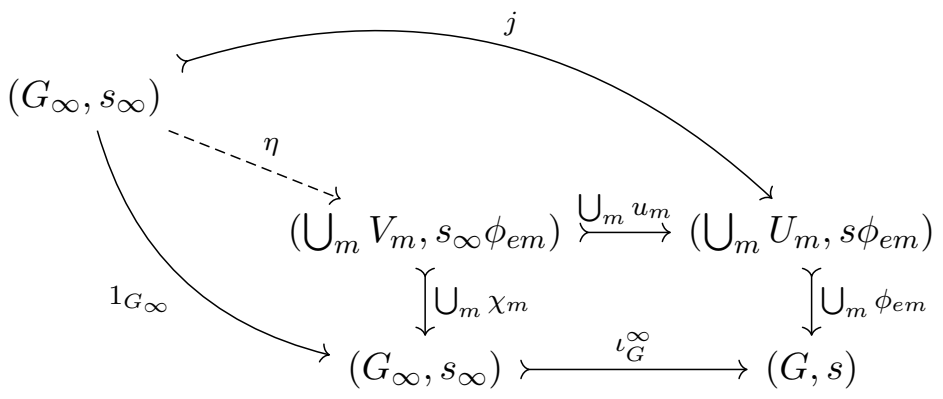

is a pullback. But then the arrow $\eta$ induced by the pair $\left(1_{G_{\infty}}, j\right)$ is an isomorphism and hence $\bigcup_{m \in M} \chi_{m}$ is a cover of $\left(G_{\infty}, s_{\infty}\right)$.

\subsection{Jet part of a Lie groupoid is internal path connected}

Now we combine Section 3.4 and Section 3.5 to show that the jet part of a Lie groupoid is $\mathcal{E}$-path connected. It will suffice to show that when we restrict the fillers $\psi_{e m}: C^{n+k} \rightarrow \mathbb{G}^{\mathbb{I}}$ defined in Section 3.4 along $u_{m}$ we get an arrow that factors through $\left(\mathbb{G}_{\infty}^{\mathbb{I}}, s_{\infty}\right)$. Then $\psi_{e m} u_{m}$ is a filler for $\chi_{m}$.

So let $V_{m}$ and $W_{m}$ be defined by the iterated pullback:

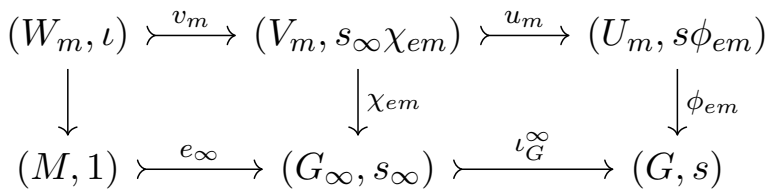


and note that the $\chi_{e m}$ are Penon open because the $\phi_{e m}$ are. Then by Proposition 3.6 and Lemma 3.9 we deduce that $v_{m}$ is jet dense. Since we have chosen $\psi_{e m}$ such that $\psi_{e m} v_{m} u_{m}$ are the constant functions $c_{m}$ the square

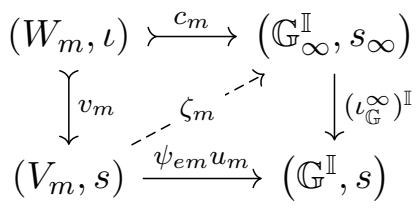

commutes and has a unique filler. This means that the $\phi_{e m}$ form a Penon open cover of $G_{\infty}$ whose fillers factor through $\mathbb{G}_{\infty}^{\mathbb{I}}$. By pulling back this cover along generalised elements $X \rightarrow G_{\infty}$ we deduce that the jet part $\mathbb{G}_{\infty}$ is $\mathcal{E}$-path connected.

\section{Integral completeness}

One of the main assumptions that we require to prove Lie's second theorem in [5] is that of integral completeness. Recall from Definition 1.9 that an arbitrary groupoid $\mathbb{G}$ in a well-adapted model $\mathcal{E}$ of synthetic differential geometry is integral complete iff

$$
\mathbb{G}^{\mathbb{I}} \stackrel{\mathbb{G}^{L_{1}}}{\longrightarrow} \mathbb{G}^{\mathbb{I} \infty}
$$

is an isomorphism in $\operatorname{Gpd}(\mathcal{E})$ where $\mathbb{I}$ is the pair groupoid on the unit interval $I$. In Section 4.3 we show that the classical $A$-paths (see for instance [13]) correspond to global sections of $\mathbb{G}^{\mathbb{I}}$ in $\mathcal{E}$ and the classical $G$-paths (see also [13]) correspond to global sections of $\mathbb{G}^{\mathbb{I}}$ in $\mathcal{E}$. In Section 4.5 we show that all classical Lie groupoids are integral complete. But first we give a more explicit description of the arrow space of $\mathbb{I}_{\infty}$.

\subsection{Representing object for infinitesimal paths is trivial}

In this section we show that the arrow space $\mathbb{I}_{\infty}^{2}$ of $\mathbb{I}_{\infty}$ is isomorphic to $I \times D_{\infty}$.

Recall from Lemma 3.3 that the arrow space of $\mathbb{I}_{\infty}$ is characterised as follows. A generalised element $(a, b) \in\left(I^{2}, \pi_{1}\right)$ is in $\left(\mathbb{I}_{\infty}^{2}, \pi_{1}\right)$ iff there exists $m \in\left(I, 1_{I}\right)$ such that $(m, m) \approx(a, b)$. By definition of $\approx$ if $b-a \in D_{\infty}$ then $a \approx b$. This means that it will suffice to prove the following result:

Lemma 4.1. If $(a, b): X \rightarrow I^{2}$ and $a \approx b$ in $\mathcal{E}$ then $b-a \in D_{\infty}$.

Proof. First suppose that $a \sim b$. This means that there exist $W \in \operatorname{Spec}\left(\right.$ Weil), $\phi \in I^{D_{W}}$ and $d \in D_{W}$ such that $\phi(0)=a$ and $\phi(d)=b$. Then by the Kock-Lawvere axiom $b=a+N$ for some nilpotent $N$.

Suppose now that $a \approx b$. This means that there exist $a_{0}, \ldots, a_{n}$ such that $a=a_{0} \sim a_{1} \sim \cdots \sim$ $a_{n}=b$. Now we know that for all $i \in\{1, \ldots, n\}$ there exists $k_{i} \in \mathbb{N}$ such that $\left(a_{i}-a_{i-1}\right)^{k_{i}}=0$. But then $(b-a)^{\Sigma_{i} k_{i}}=0$ as required.

Corollary 4.2. The groupoid $\mathbb{I}_{\infty}$ has underlying reflexive graph isomorphic to

$$
I \times D_{\infty} \underset{\pi_{1}}{\stackrel{+}{\rightleftarrows}} I,
$$

where $e=\left(1_{I}, 0\right)$ and composition $I \times D_{\infty} \times D_{\infty} \rightarrow I \times D_{\infty}$ defined by

$$
\left(a, d, d^{\prime}\right) \mapsto\left(a, d+d^{\prime}\right) .
$$

Proof. For all $a \in I$ we have $a \approx a+d$ and we can define an arrow $I \times D_{\infty} \rightarrow \mathbb{I}_{\infty}^{\mathbf{2}}$ by $(a, d) \mapsto(a, a+d)$. The inverse $(a, b) \mapsto(a, b-a)$ factors through $I \times D_{\infty}$ by Lemma 4.1. 


\subsection{Formal group laws}

When we form the infinitesimal part of a category in [5] our construction corresponds to the part of a Lie group represented by its formal group law. Following [14] we define an $n$-dimensional formal group law $F$ to be an $n$-tuple of power series in the variables $X_{1}, \ldots, X_{n} ; Y_{1}, \ldots, Y_{n}$ with coefficients in $\mathbb{R}$ such that the equalities

$$
F(\vec{X}, \overrightarrow{0})=\vec{X}, \quad F(\overrightarrow{0}, \vec{Y})=\vec{Y} \quad \text { and } \quad F(F(\vec{X}, \vec{Y}), \vec{Z})=F(\vec{X}, F(\vec{Y}, \vec{Z}))
$$

hold. We refer to the Introduction of [14] for the construction of a formal group law from a Lie group. In fact the category of Lie algebras and formal group laws are shown to be equivalent in Theorem 3 of Section V.6 of Part 2 in [26].

In the following example we show how to reformulate the construction of a formal group law from a Lie group in terms of the infinitesimal elements of the Lie group.

Example 4.3. Let $(G, \mu)$ be a Lie group whose underlying smooth manifold is $n$-dimensional. Since $G$ is locally isomorphic to $R^{n}$ we see that its jet part is a group of the form $\left(D_{\infty}^{n}, \mu\right)$ by a straightforward extension of Lemma 4.1. Now to give a multiplication

$$
\mu: D_{\infty}^{n} \times D_{\infty}^{n} \rightarrow D_{\infty}^{n}
$$

is to give arrows

$$
f_{1}, \ldots, f_{n}:\left(D_{\infty}\right)^{2 n} \rightarrow R
$$

taking values in nilpotent elements. Now we have that

$$
\left(D_{\infty}\right)^{2 n}=\bigcup_{k}\left(D_{k}\right)^{2 n}
$$

and so, since $\mathcal{E}(-, R)$ sends colimits to limits the hom-set $\mathcal{E}\left(D_{\infty}^{2 n}, R\right)$ is given by the limit

$$
\cdots \rightarrow \mathcal{E}\left(D_{k+1}^{2 n}, R\right) \rightarrow \mathcal{E}\left(D_{k}^{2 n}, R\right) \rightarrow \cdots,
$$

which by the Kock-Lawvere axiom is equivalently the limit of the polynomial algebras

$$
\cdots \rightarrow \mathbb{R}\left[X_{1}, \ldots, X_{2 n}\right] / I_{k+1} \rightarrow \mathbb{R}\left[X_{1}, \ldots, X_{2 n}\right] / I_{k} \rightarrow \cdots
$$

where $I_{k}$ is the ideal generated by $\left(X_{1}^{k}, X_{2}^{k}, \ldots, X_{2 n}^{k}\right)$. This means that $\mathcal{E}\left(D_{\infty}^{2 n}, R\right)$ can be identified with the ring $\mathbb{R}\left[\left[X_{1}, \ldots, X_{2 n}\right]\right]$ of formal power series. Now the condition that the $f_{i}$ take values in the nilpotent elements implies that the constant term of the power series $p_{i}$ corresponding to $f_{i}$ is zero. Under this correspondence, the group axioms for $G$ correspond to the axioms making $p_{1}, \ldots, p_{n}$ into a formal group law.

\subsection{Paths of infinitesimals}

The correct notion of a path of infinitesimal arrows in a Lie groupoid $\mathbb{G}$ is that of an $A$-path (see for instance [6]). In the topos $\mathcal{E}$ the object of $A$-paths $A(\mathbb{G})$ associated to $\mathbb{G}$ is the subobject of all $\phi \in G^{I \times D}$ such that for all $a \in I$ and all $d \in D$ the arrows $\phi(a, 0)$ are identity arrows, the $\phi(a,-)$ are source constant and $t \phi(a, d)=t \phi(a+d, 0)$. Note that since $G^{D} \cong T G$ the global sections of $A(\mathbb{G})$ are precisely the $A$-paths defined in Section 1 of [6].

In this section we show that $A(\mathbb{G}) \cong \mathbb{G}^{\mathbb{I} \infty}$ in $\mathcal{E}$ where $\mathbb{I}_{\infty}$ is the jet part of the pair groupoid $\mathbb{I}$ on the unit interval $I$. Using Corollary 4.2 we see that $\mathbb{G}^{\mathbb{I}_{\infty}}$ is the subobject of all $\phi \in G^{I \times D_{\infty}}$ 
such that for all $a \in I$ and all $d \in D_{\infty}$ the arrows $\phi(a, 0)$ are identity arrows, the $\phi(a,-)$ are source constant and not only does $t \phi(a, d)=t \phi(a+d, 0)$ hold but indeed

$$
\phi\left(a, d+d^{\prime}\right)=\phi\left(a+d, d^{\prime}\right) \phi(a, d)
$$

holds for all $d, d^{\prime} \in D_{\infty}$. This means that there is a natural restriction arrow $\mathbb{G}^{\mathbb{I} \infty} \rightarrow A(\mathbb{G})$. In this section we describe its inverse.

To do this we define an arrow $v: G^{I \times D} \rightarrow G^{I \times D_{\infty}}$ which satisfies $v(\phi)\left(a, d+d^{\prime}\right)=v(\phi)(a+$ $\left.d, d^{\prime}\right) v(\phi)(a, d)$ for all $d, d^{\prime} \in D_{\infty}$. Recall that $D_{\infty}=\bigcup_{i} D_{i}$ and so it will suffice to find for all $i \in \mathbb{N}$ an arrow $v_{i}: G^{I \times D} \rightarrow G^{I \times D_{i}}$ such that $v_{i+j}(\phi)\left(a, d+d^{\prime}\right)=v_{j}(\phi)\left(a+d, d^{\prime}\right) v_{i}(\phi)(a, d)$ for all $d \in D_{i}$ and $d^{\prime} \in D_{j}$.

Now we recall the following slight generalisation of the Bunge axiom that is Proposition 4 in Section 2.3.2 in [19]:

Lemma 4.4. Let $i \in \mathbb{N}$ and consider the arrows $f_{1}, \ldots, f_{i}: D^{i-1} \rightarrow D^{i}$ defined by

$$
f_{m}\left(d_{1}, \ldots, d_{i-1}\right)=\left(d_{1}, \ldots, d_{m-1}, 0, d_{m}, \ldots, d_{i-1}\right) .
$$

Then for any microlinear space $G$ the arrow

$$
G^{D_{i}} \stackrel{G^{+}}{\longrightarrow} G^{D^{i}}
$$

is the joint equaliser of $G^{f_{1}}, \ldots, G^{f_{i}}$.

Using Lemma 4.4 we see that it will now suffice to find for all $i \in \mathbb{N}$ an arrow $v_{i}: G^{I \times D} \rightarrow$ $G^{I \times D^{i}}$ such that for all $m, l \in\{1, \ldots, i\}$ the equalities $G^{I \times f_{m}} v_{i}(\phi)=G^{I \times f_{l}} v_{i}(\phi)$ and

$$
v_{i+j}(\phi)\left(a,\left(d_{1}, \ldots, d_{i+j}\right)\right)=v_{j}(\phi)\left(a+\sum_{m=1}^{i} d_{m},\left(d_{i+1}, \ldots, d_{i+j}\right)\right) v_{i}(\phi)\left(a,\left(d_{1}, \ldots, d_{i}\right)\right)
$$

hold for the $f_{i}$ defined in Lemma 4.4 .

Lemma 4.5. The restriction $\mathbb{G}^{\mathbb{I}_{\infty}} \rightarrow A(\mathbb{G})$ has an inverse.

Proof. The arrows $v_{i}: G^{I \times D} \rightarrow G^{I \times D^{i}}$ defined by

$$
v_{i}(\phi)\left(a,\left(d_{1}, \ldots, d_{i}\right)\right)=\phi\left(a+\Sigma_{m=1}^{i-1} d_{m}, d_{i}\right) \cdots \phi\left(a+d_{1}, d_{2}\right) \phi\left(a, d_{1}\right)
$$

satisfy $G^{I \times f_{m}} v_{i}(\phi)=G^{I \times f_{l}} v_{i}(\phi)$ because $\phi(a, 0)$ are identity arrows for all $a \in I$ and satisfies

$$
v_{i+j}(\phi)\left(a,\left(d_{1}, \ldots, d_{i+j}\right)\right)=v_{j}(\phi)\left(a+\sum_{m=1}^{i} d_{m},\left(d_{i+1}, \ldots, d_{i+j}\right)\right) v_{i}(\phi)\left(a,\left(d_{1}, \ldots, d_{i}\right)\right)
$$

by construction. It is easy to see that the $v_{i}$ define an inverse to the restriction.

\subsection{Integration of paths of infinitesimals is groupoid enriched}

Recall that in Definition 1.9 we defined the notion of integral complete groupoid using an isomorphism in the category $\operatorname{Gpd}(\mathcal{E})$. The following result show that we only need to check this condition on the space of objects which is an object of $\mathcal{E}$.

Proposition 4.6. If $\mathbb{G}^{\iota_{\infty}}: \mathbb{G}^{\mathbb{I}} \rightarrow \mathbb{G}^{\mathbb{I} \infty}$ is an isomorphism in a well-adapted model $\mathcal{E}$ then it is an isomorphism of groupoids also. 
Proof. We need to show that natural transformations extend uniquely, i.e.,

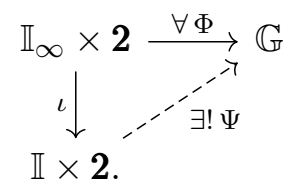

Let $\psi_{0}, \psi_{1}$ be the unique lifts of $\phi$ precomposed with the two inclusions of 1 into 2 . If for all $x \rightarrow y$ in $\mathbb{I}$ the diagram

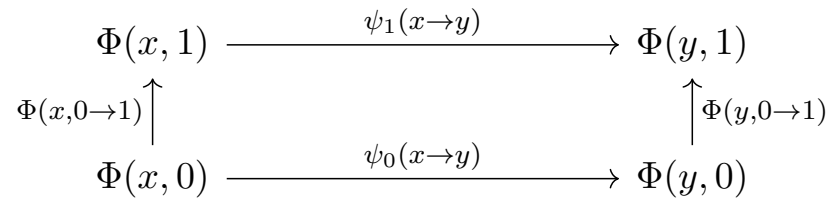

commutes then we can define $\Psi(x \rightarrow y, 0 \rightarrow 1)$ to be this common value. To this end define $\theta: \mathbb{I} \rightarrow \mathbb{G}$ to take $x \rightarrow y$ to

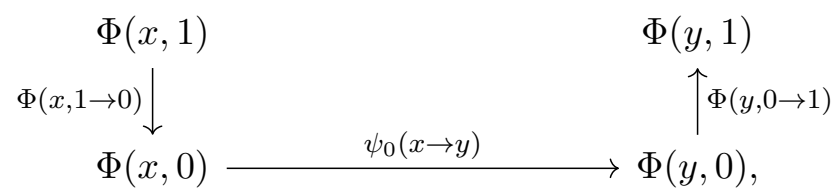

when we restrict to $\mathbb{I}_{\infty}$ (i.e., take $y=x+d$ ) we see that

$$
\begin{gathered}
\Phi(x, 1) \underset{\Phi(x+d, 0 \rightarrow 1) \uparrow}{\Phi(x+d, 1)} \\
\Phi(x, 1 \rightarrow 0) \downarrow \\
\Phi(x, 0) \stackrel{\Phi(x \rightarrow x+d, 0)}{\longrightarrow} \Phi(x+d, 0)
\end{gathered}
$$

and so by the uniqueness of lifts $\theta=\psi_{1}$ and (4.2) commutes.

\subsection{Lie groupoids are integral complete}

We show that $\mathbb{G}_{\mathbb{I}}^{\iota^{\infty}}: \mathbb{G}^{\mathbb{I}} \rightarrow \mathbb{G}^{\mathbb{I} \infty}$ is an isomorphism in $\operatorname{Gpd}(\mathcal{E})$. By Proposition 4.6 it will suffice to show that $\mathbb{G}_{\mathbb{I}}^{\iota^{\infty}}$ is an isomorphism in $\mathcal{E}$. More concretely, we show that for all representable objects $X$ and arrows $\phi: X \rightarrow \mathbb{G}^{\mathbb{I}}$ there exists a (unique) $\psi: X \rightarrow \mathbb{G}^{\mathbb{I}}$ such that $\mathbb{G}^{\iota_{\mathbb{I}}^{\infty}} \psi=\phi$.

By Corollary 4.2 arrows $\phi: X \rightarrow \mathbb{G}^{\mathbb{I} \infty}$ correspond to arrows $\phi: X \times I \times D_{\infty} \rightarrow G$ such that $\phi(x, a, 0)$ are identity arrows and $\phi(x, a,-)$ are source constant. It is easy to see that arrows $\psi: X \rightarrow \mathbb{G}^{\mathbb{I}}$ correspond to arrows $\psi: X \times I \rightarrow G$ such that $\psi(x, 0)$ are identity arrows and $\psi(x,-)$ are source constant. At this point it is convenient to assume that the topos $\mathcal{E}$ is generated by a subcanonical site whose underlying category is a full subcategory of the category of affine $C^{\infty}$-schemes as defined in Definition 1.5. In particular this means that every representable object is a closed subset of $R^{n}$ for some $n \in \mathbb{N}$. Recall from Lemma 2.26 in [20] that if we are given a smooth function that has as domain any closed subset of $\mathbb{R}^{n}$ we can lift it to a smooth function on the whole of $\mathbb{R}^{n}$. Therefore since every representable $X$ is a closed subset of $R^{n}$ for some $n \in \mathbb{N}$ it will suffice to prove the result in the case $X=R^{n}$.

Theorem 4.7. For all $\phi: R^{n} \times I \times D_{\infty} \rightarrow G$ such that $\phi(x, a, 0)$ are identity arrows, $\phi(x, a,-)$ is source constant and $\phi\left(a, d+d^{\prime}\right)=\phi\left(a+d, d^{\prime}\right) \phi(a, d)$ for $x \in R^{n}, a \in I$ and $d, d^{\prime} \in D_{\infty}$ there exists a unique $\psi: R^{n} \times I \rightarrow G$ such that $\psi(x, 0)$ are identity arrows, $\psi(x,-)$ is source constant and $\psi(x, a+d)=\phi(x, a, d) \psi(x, a)$ for all $d \in D_{\infty}$. 
Proof. To do this we make rigorous the intuitive idea of composing together infinitely many infinitesimal arrows to get a macroscopic arrow. First let $\phi_{0}=s \phi(-,-, 0)=t \phi(-,-, 0)$. Then the pullback

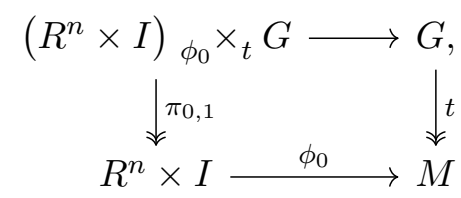

is a manifold because $t$ is a submersion. Since $\phi(x, a,-)$ is source constant the infinitesimal action

$$
\begin{aligned}
R^{n} \times D \times\left(\left(R^{n} \times I\right)_{\phi_{0}} \times{ }_{t} G\right) & \rightarrow\left(\left(R^{n} \times I\right)_{\phi_{0}} \times{ }_{t} G\right), \\
(y, d, x, a, g) & \mapsto(x, a+d, \phi(x, a, d) \circ g)
\end{aligned}
$$

defines a collection of smoothly parameterised vector fields on $\left(R^{n} \times I\right){ }_{\phi_{0}} \times{ }_{t} G$. Since the solution curves of smooth vector fields have a smooth dependence on parameters we obtain using the initial conditions $\psi(y, 0)=(y, 0, \phi(y, 0,0))$ a parameterised solution $\psi: R^{n} \times I \rightarrow\left(R^{n} \times I\right)_{\phi_{0}} \times{ }_{t} G$ which satisfies:

- $\psi(y, 0)=\left(\psi_{1}(y, 0), \psi_{2}(y, 0), \psi_{3}(y, 0)\right)=(y, 0, \phi(y, 0,0))$,

- $\psi_{1}(y, a+d)=\psi_{1}(y, a)$,

- $\psi_{2}(y, a+d)=\psi_{2}(y, a)+d$,

- $\psi_{3}(y, a+d)=\phi\left(\psi_{1}(y, a), \psi_{2}(y, a), d\right) \circ \psi_{3}(y, a)$.

Therefore

- $\psi_{1}(y, a)=\psi_{1}(y, 0)=y$,

- $\psi_{2}(y, a)=\psi_{2}(y, 0)+a=a$,

- $\left.\psi_{3}(y, a+d)=\phi\left(\psi_{1}(y, a), \psi_{2}(y, a), d\right) \psi_{3}(y, a)=\phi(y, a, d) \circ \psi_{3}(y, a)\right)$,

and $\psi_{3}$ is the map we require. Now we check that $\psi_{3}$ does indeed define a $G$-path. The map $\psi_{3}$ is source constant in the second variable because

$$
s \psi_{3}(y, a+d)=s(\phi(y, a, d) \circ \psi(x, a))=s \psi_{3}(y, a)
$$

for all $d \in D$. Finally we appeal to Proposition 2.7 in [18] to conclude that $\psi_{3}(y, a+d)=$ $\phi(y, a, d) \psi_{3}(y, a)$ holds for all $d \in D_{\infty}$.

\section{Acknowledgements}

The author is very grateful for the constructive comments offered by and the important corrections indicated by the editor and referees. The author would like to acknowledge the assistance of Richard Garner, my Ph.D. supervisor at Macquarie University Sydney, who provided valuable comments and insightful discussions in the genesis of this work. In addition the author is grateful for the support of an International Macquarie University Research Excellence Scholarship. 


\section{References}

[1] Almeida R., Molino P., Suites d'Atiyah et feuilletages transversalement complets, C. R. Acad. Sci. Paris Sér. I Math. 300 (1985), 13-15.

[2] Bruno O.P., Logical opens of exponential objects, Cahiers Topologie Géom. Différentielle Catég. 26 (1985), $311-323$.

[3] Bunge M., Dubuc E.J., Local concepts in synthetic differential geometry and germ representability, in Mathematical Logic and Theoretical Computer Science (College Park, Md., 1984-1985), Lecture Notes in Pure and Appl. Math., Vol. 106, Dekker, New York, 1987, 93-159.

[4] Burke M., Synthetic Lie theory, Ph.D. thesis, Macquarie University, Sydney, 2015, available at http://hdl . handle.net/1959.14/1068209.

[5] Burke M., A synthetic version of Lie's second theorem, arXiv:1605.06378.

[6] Crainic M., Fernandes R.L., Integrability of Lie brackets, Ann. of Math. 157 (2003), 575-620, math.DG/0210152.

[7] del Hoyo. Matias, Fernandes R.L., Riemannian metrics on Lie groupoids, J. Reine Angew. Math., to appear, arXiv:1404.5989.

[8] Douady A., Lazard M., Espaces fibrés en algèbres de Lie et en groupes, Invent. Math. 1 (1966), $133-151$.

[9] Dubuc E.J., Sur les modèles de la géométrie différentielle synthétique, Cahiers Topologie Géom. Différentielle 20 (1979), 231-279.

[10] Dubuc E.J., $C^{\infty}$-schemes, Amer. J. Math. 103 (1981), 683-690.

[11] Dubuc E.J., Logical opens and real numbers in topoi, J. Pure Appl. Algebra 43 (1986), 129-143.

[12] Dubuc E.J., Germ representability and local integration of vector fields in a well adapted model of SDG, J. Pure Appl. Algebra 64 (1990), 131-144.

[13] Duistermaat J.J., Kolk J.A.C., Lie groups, Universitext, Springer-Verlag, Berlin, 2000.

[14] Hazewinkel M., Formal groups and applications, Pure and Applied Mathematics, Vol. 78, Academic Press, Inc., New York - London, 1978.

[15] Hirsch M.W., Differential topology, Graduate Texts in Mathematics, Vol. 33, Springer-Verlag, New York Heidelberg, 1976.

[16] Johnstone P.T., Sketches of an elephant: a topos theory compendium, Vol. 3, privately communicated preprint.

[17] Kock A., Synthetic differential geometry, London Mathematical Society Lecture Note Series, Vol. 333, 2nd ed., Cambridge University Press, Cambridge, 2006.

[18] Kock A., Reyes G.E., Ordinary differential equations and their exponentials, Cent. Eur. J. Math. 4 (2006), 64-81.

[19] Lavendhomme R., Basic concepts of synthetic differential geometry, Kluwer Texts in the Mathematical Sciences, Vol. 13, Kluwer Academic Publishers Group, Dordrecht, 1996.

[20] Lee J.M., Introduction to smooth manifolds, Graduate Texts in Mathematics, Vol. 218, 2nd ed., Springer, New York, 2013.

[21] Mackenzie K.C.H., General theory of Lie groupoids and Lie algebroids, London Mathematical Society Lecture Note Series, Vol. 213, Cambridge University Press, Cambridge, 2005.

[22] Michor P.W., Topics in differential geometry, Graduate Studies in Mathematics, Vol. 93, Amer. Math. Soc., Providence, RI, 2008.

[23] Moerdijk I., Reyes G.E., Models for smooth infinitesimal analysis, Springer-Verlag, New York, 1991.

[24] Penon J., Infinitésimaux et intuitionnisme, Cahiers Topologie Géom. Différentielle 22 (1981), 67-72.

[25] Penon J., De l'infinitésimal au local, Diagrammes 13 (1985), iv+191 pages.

[26] Serre J.-P., Lie algebras and Lie groups, Lecture Notes in Math., Vol. 1500, Springer-Verlag, Berlin, 2006.

[27] Tseng H.-H., Zhu C., Integrating Lie algebroids via stacks, Compos. Math. 142 (2006), 251-270, math.DG/0405003. 